\title{
Structural Innovation Design of the Compressor Impeller
}

\author{
Xiaomei You ${ }^{1, a}$, Lei Meng ${ }^{2, b}$, Bangchun Wen ${ }^{3, c}$ \\ ${ }^{1}$ School of Mechanical Engineering, ShenYang LiGong University, 6 NanPing middle \\ Road ,Shenyang, China ,110159 \\ ${ }^{2}$ SBW Turbo Machinery Corporation, 16 KaiFa Road, Shenyang, China,110869 \\ ${ }^{3}$ School of Mechanical Engineering and Automation, Northeastern University, 3 WenHua \\ Road ,Shenyang, China, 110004 \\ aprime_queen@163.com, ${ }^{\mathrm{b}} 1080907 @ q q . c o m,{ }^{\mathrm{c}}$ bcwen1930@sina.vip.com
}

\begin{abstract}
Keywords:product innovative design; impeller; the product integrated design theory and method; viewable optimization design method

Abstract. For the main problems in practical applications of the impeller (key part of the large-scale centrifugal compressor), based on the product integrated design theory, the design quality model of the impeller overall considered its structural performance and manufacturing performance is built, and the viewable optimization design method is used to complete the structure innovation of the new impeller. Accordingly to the design method above, reference is provided for the innovative design of the other key components of machinery products.
\end{abstract}

\section{Introduction}

As the key component of the rotor system of centrifugal compressor, property of the impeller has a direct effect on the normal operation of the compressor. Working Principle of the conventional impeller and its main problems in practical applications are shown in literature[1].Now, as the performance of centrifugal compressor develops towards large flow rate,high speed etc., meeting the new demands of the impeller for large performance range of operating conditions,manufacturing easily etc. has becoming a key issue.

In the traditional design, the structural optimization design methods of the impeller are usually discussed just from the point of view of the structure, or the strength. Methods above are usually applied to design the conventional impeller. However, the innovative design quality of some unconventional, non-standard impellers can not be guaranteed by the design methods above. So based on the product integrated design theory put forward by professor Wen Bangchun, the design quality model of the impeller overall considered its structural performance and manufacturing performance is built, and then the viewable optimization design method is used to complete the structure innovation of the new impeller.

\section{Building the design quality model of the impeller}

Impeller's function is determined by the compressor requirements. Before designing the impeller, data of the blade profile meeting requirements of the aerodynamic performance will be often given, then the meridional flow line can be determined. Then the impeller's function is determined, so its design quality is mainly reflected in the performance. Among them, the structural performance and manufacturing performance are the two key quality components in the innovative design phase. Accordingly to the product integrated design theory[2], the design quality model of the impeller built is shown in fig.1.

In the innovative design process of the impeller structure, the main consideration of the structure properties include strength properties, deformation trend, shaft-hole displacement, and the dynamic characteristics in the movement conditions; and also the main consideration of the manufacturing properties include processing performance 、 welding performance and assembly process performance. 


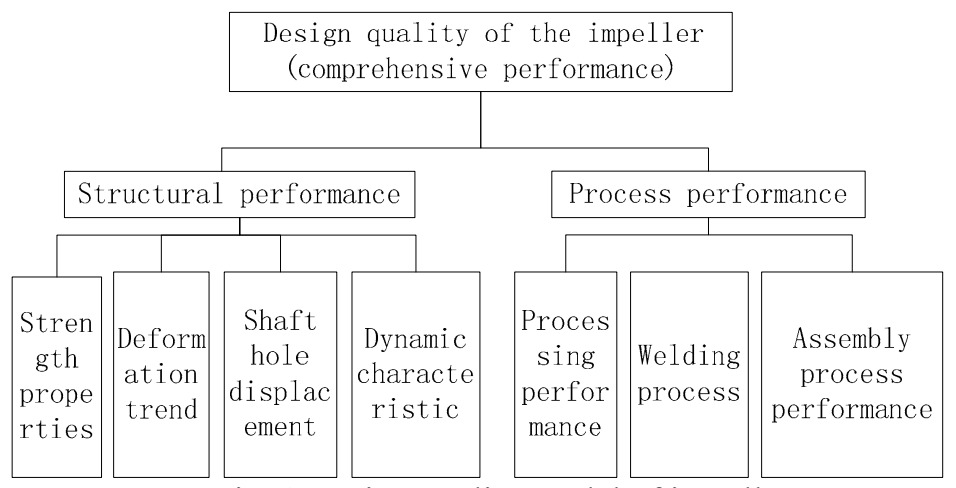

Fig. 1 Design quality model of impeller

\section{Designing the Initial Structure of the New Impeller}

Determining the Design Space. Before designing the new impeller, its shape of the flow channel is often determined(by the flow data given). Because it is a new structural and there has not previous experience, determining the design space is the primary task. In the determining process , the visual optimization design method is used to describe the design space qualitatively and intuitively from space structure, welding process, assembly process and the processing performance.

Limiting Condition of the Structure Space. Maximum sizes of cover-disk and shaft -disk are restricted by design size and location of other pneumatic originals in the serialization process of subsequent products. The envelope space formed by these pneumatic originals is the structure space of the new impeller, and its visual specific location is shown in the blue area of fig. $2 \mathrm{a} \sim \mathrm{f}$.

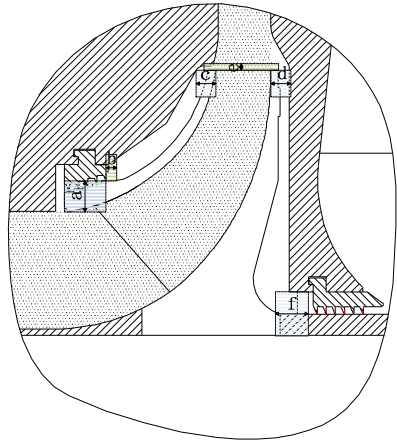

Fig.2 Structure space limit of the new impeller

From the figure, it can be seen that the imports thickness limit to the cover-disk because of seal locations(corresponding to the size a)、 the limit to the width of the mouth ring of the cove-disk because of seal processing platform positions(corresponding to the size $b$ )、 the limit to the thickness of cover-disk and shaft-disk because of the diffuser entrance width(corresponding to the size $c 、 d$ and e) and the limit to the length and thickness of the impeller tail because of the interstage seal positions are all considered (corresponding to the size f).All these locations above are given by designers considering comprehensively the factors such as the axial momentum of the rotor、 shape variables of the impeller and baffles in the rotation and pressure condition 、 the thermal expansion and the vibration of the rotor during operation.

Considering Welding Performance. The line-element blade is used in design. In the entrance of blades, the angle between the blade and the blade-disk is large, and the cascade is more dense, which are easy to result in the visual blind spot in the welding process. So there is possibility of weld failure. Using traditional design methods, it will usually be ignored. In this paper, the visual optimization design method is used to establish the visual model in establishing initial structure programs and to judge intuitively through visual indicators, and then to optimize the design program so as to avoid the repetitive design. So in designing, the width of the mouth ring is truncated reasonably to reduce the operation difficulty of welding operators and to reduce the 
welded dead-angle at the cover-disk. The specific implementation effect is shown in figure 3.

Considering Processing Performance. In order to reduce weight in the subsequent balancing process, the mass removing structure of the shaft-disk dorsal of the impeller is explicitly designed.

After preliminary analysis, it is found that the boss used to remove mass is more close to the rim, stress of the impeller is better to improve. However, if the boss is fully applied in the rim, detecting the beat value is difficulty in machining process. So, the boss is determined to be $10 \mathrm{~mm}$ from the rim according to the technical operations specifications.

Considering Technology Installation Performance. Wheels of the new impeller is designed to be longer than that of the conventional impeller, so the contact area between the wheels and the shaft is also greater, which will increase the difficulty of assembling in the same interference conditions. At the same time, increasing the contact area between the wheels and the shaft will make the structural performance uncertain in the tooling process of the shaft. So, in the design process, innovation of the hub structure is an important content of the research work.

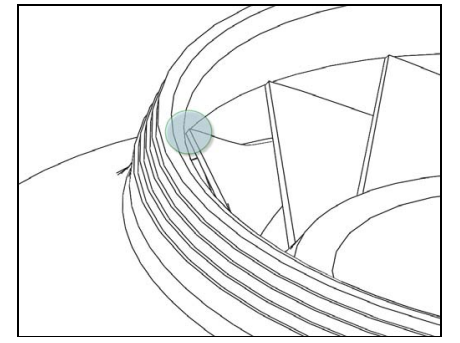

a. the original design structure

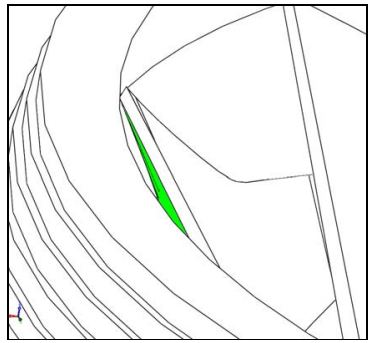

b. the optimal design structure

Fig. 3 Visual range comparison between primitive design structure and optimization design structure

\section{Establishing the Visual Program of Initial Structure of the Impeller}

Accordingly to the method of determining the design space above and based on the flow data given, the initial structure program of the new impeller can be determined, which is shown in figure 4.

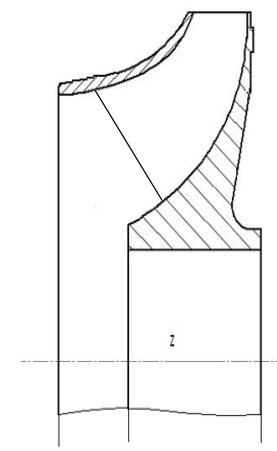

Fig. 4 Structure initial scheme of the new impeller

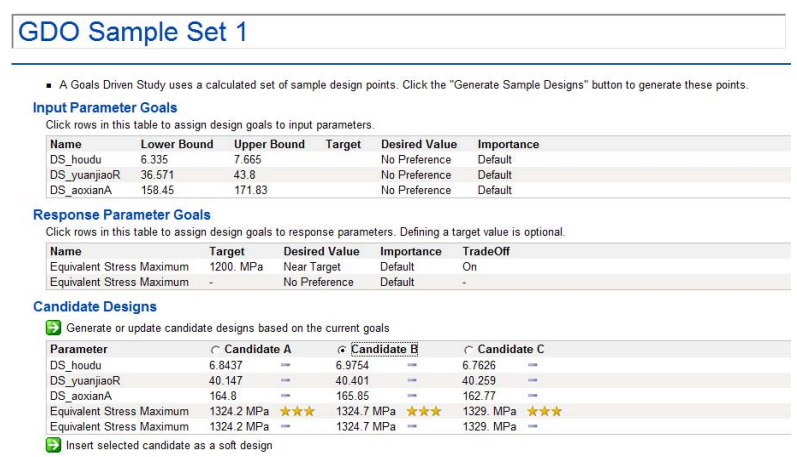

Fig. 5 Optimization result of key parameters

In order to determine the specific structure of the new impeller, the visual optimization design method is used to analyze mechanical properties qualitatively on the initial structure program, and to study structural parameters impacting mechanical properties of it, while combined with relevant experience on structure optimization design, the key structural parameters impacting on mechanical properties of the impeller are determined.

\section{Designing the Specific Structure Program of the New Impeller}

Sensitivity analysis and optimization design of structural parameters. According to the visual analysis results of the initial program, key parameters are planned. Then the outer edge thickness of the shaft-disk , depression fillet size and depression dip of the shaft-disk dorsal are determined to be the main structural parameters impacting the comprehensive performance. Based on ANSYS, sensitivity analysis is done to study how the structural parameters effects on 
mechanical properties. System optimization result in 10000 iterations calculation conditions is shown in fig,5.

From the figure it can be seen that, in the iteration process, Misses stress of the impeller is the lowest when the thickness of the shaft-disk is 7, depression fillet radius of the shaft-disk is 40 and the reverse angle between depression back-disk and the $\mathrm{X}$-axis is $165^{\circ}$. From those above, shaped line size of back-disk is basically determined. Sensitivity analysis results are shown in fig. 6 .

It can be seen from figure 6 that in the inertia force, maximum stress of the impeller is effected greatly by the dip of the shaft-disk dorsal and the outer edge thickness of the shaft-disk, so these two structural parameters are key parameters in the impeller structure optimization process. Accordingly to sensitivity analysis results of structure parameters, the size of the initial structure of the new impeller are modified. The visual structure of the new impeller after the optimum design is shown in fig. 7.
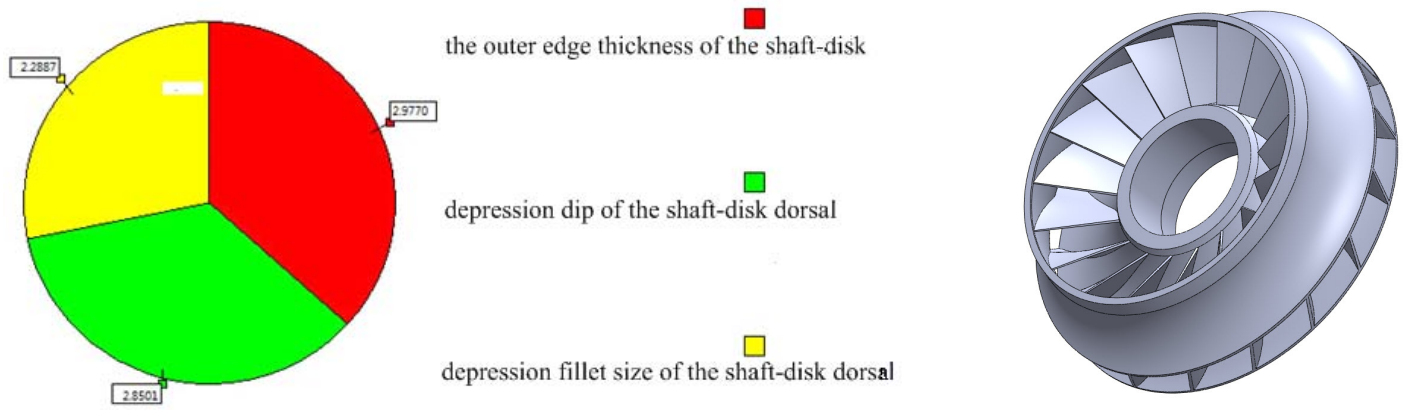

Fig. 6 Sensitivity analysis pie-chart of structure parameters

Fig. 7 Concrete structure of the new impeller

\section{Conclusions}

Based on the product integrated design theory, For the main problems in practical applications of the impeller (the key part of large-scale centrifugal compressors), the structural innovation design method oriented comprehensive performance of the impeller is put forward. The design quality model of the impeller is built, and the viewable optimization design method is used to complete the structure innovation of the new impeller. The design method put forward in this paper provides a new way of thinking and means for innovatively designing other key parts of mechanical products.

\section{Acknowledgements}

This work was financially supported by the National Natural Science Foundation (50535010).

This work was financially supported by the Research project funded by Education Department of Liaoning Province (L2010477).

\section{References}

[1] You XiaoMei. Research on Innovation and its Application of Every Deign Stages of Product Based on Scientific Outlook on Development [D]. Northeastern University Press, 2011

[2] Wen BangChun. Integrated design of product full function and overall performance [M].China Machine Press, 2008

[3] MENG Lei. General quality-oriented $1+3+X$ designing method of the assembly structure of the shrouded impeller[D]. Master's Degree thesis of Northeastern University,2007

[4] SHU Xin-wei; GU Chuan-gang; WANG Tonga method for the optimized design of centrifugal compressor Blades[J], Journal of Engineering for Thermal Energy and Power, 2008, 23(2).

[5] GUAN ZhenQun, LUO YangJun, WANG XueJun etc. shape optimization method of centrifugal impeller based-on 3D parameterization[J], Journal of Mechanical Strength,2006,28(6). 